Trans

continentales
Transcontinentales

Sociétés, idéologies, système mondial

5 | 2007

Le défi sanitaire

\title{
Droits de propriété intellectuelle et santé publique dans les pays du Sud
}

Intellectual Property Rights and Public Health in Southern Hemisphere

Countries: The Anti-AIDS Drugs Issue

\section{Samira Guennif}

\section{CpenEdition}

Journals

Édition électronique

URL : http://journals.openedition.org/transcontinentales/682

DOI : 10.4000/transcontinentales.682

ISBN : 978-2-7351-1563-1

ISSN : 1775-397X

Éditeur

Editions de la maison des sciences de l'homme

Édition imprimée

Date de publication : 31 décembre 2007

Pagination : 19-37

ISBN : 978-2-200-92397-6

ISSN : 1950-1684

Référence électronique

Samira Guennif, «Droits de propriété intellectuelle et santé publique dans les pays du Sud », Transcontinentales [En ligne], 5 | 2007, document 2, mis en ligne le 21 avril 2011, consulté le 08 septembre 2020. URL : http://journals.openedition.org/transcontinentales/682 ; DOI : https://doi.org/ $10.4000 /$ transcontinentales.682 


\title{
Droits de propriété intellectuelle et santé publique dans les pays du Sud
}

\author{
L'enjeu des médicaments anti-sida
}

\section{Samira Guennif}

Parmi les dernières résolutions prises par les instances internationales en faveur du développement socioéconomique des pays du Sud figure l'initiative onusienne. En 2000, cent quatre-vingt-onze États membres des Nations unies ont adopté la déclaration du Millénaire et se sont engagés à atteindre d'ici 2015 huit objectifs essentiels, les objectifs de développement du Millénaire (ODM). Ces objectifs ont vocation à améliorer les conditions économiques, alimentaires, sanitaires, éducatives et environnementales dans les pays en développement.

Qu'il s'agisse de combattre le VIH/sida, le paludisme et d'autres maladies, d'améliorer la santé maternelle, de réduire la mortalité des enfants de moins de 5 ans, de réduire l'extrême pauvreté et la faim, d'assurer l'éducation primaire pour tous ou encore de mettre en place un partenariat mondial pour le développement, il apparaît que la plupart des ODM ont à voir directement ou indirectement avec la sécurité sanitaire des populations. En témoignent les études évaluant les effets de l'épidémie du VIH/sida sur le développement socioéconomique des pays du Sud.

En effet, avec près de 40 millions de personnes infectées par le VIH/sida essentiellement situées dans les pays du Sud ${ }^{1}$, on observe des effets micro et macroéconomiques en chaîne qui viennent compromettre le développement socioéconomique de ces pays, déjà fragilisés par ailleurs. En bout de chaîne, les pays durement frappés par le VIH/sida subissent une réduction de l'espérance de vie des populations et une baisse de 0,8 à $1,4 \% \mathrm{du} \mathrm{PIB}^{2}$. Aussi, freiner la progression

1 - ONUSIDA, Rapport sur l'épidémie mondiale de sida, édition spéciale pour le $10^{e}$ anniversaire de l'ONUSIDA, ONUSIDA/ Programme commun des Nations unies sur le VIH/sida, 2006.

2 - N. Drouhin, V. Touzé et B. Ventelou, "Aids and economic growth in Africa : a critical assessment of the "base-case scenario" approach", in J. P. Moatti, B. Coriat, Y. Barnett, Y. Souteyrand, J. Dumoulin et P.Y. Flori (éds), Economics of Aids and Access to HIV/AIDS Care in Developing Countries. Issues and Challenges, Paris, ANRS (Sciences sociales et Sida), 2003, p. 383-413. 
de l'épidémie du VIH/sida et mettre les personnes infectées sous traitements antirétroviraux constituent des enjeux pour prévenir une dégradation des processus de développement au Sud.

Atteindre ces objectifs suppose pour l'ONU et les pays du Sud de travailler dans le sens d'une meilleure accessibilité des médicaments essentiels tels que les traitements anti-sida. Mais on doit s'interroger alors sur l'enjeu sanitaire que constitue la ratification de l'accord sur les aspects des droits de propriété intellectuelle touchant au commerce (ADPIC) et la mise en place progressive de régimes de droits de propriété intellectuelle (DPI) plus contraignants. Aussi, cet article propose de préciser les possibilités offertes aux pays en développement de promouvoir la santé publique et l'accès aux médicaments anti-sida sous renforcement de la gouvernance globale de la propriété intellectuelle.

Pour ce faire, un exposé de l'ADPIC permettra de préciser les obligations légales imposées aux pays membres de l'Organisation mondiale du commerce (OMC) en matière de protection du brevet et de lister les flexibilités prévues pour promouvoir la santé publique. Ensuite seront décrits les moyens déployés et les obstacles rencontrés par le Brésil, la Thaïlande et l'Inde pour utiliser ces flexibilités et améliorer l'accès aux médicaments essentiels.

\section{La protection du brevet : obligations générales et exceptions}

Depuis quelques années, il existe en matière de santé une tension croissante entre les pays du Nord et les pays du Sud. Les uns travaillent à une promotion soutenue des brevets dans le monde tandis que les autres déploient des ressources considérables pour promouvoir la santé publique. Ces tensions donnent régulièrement lieu à des diatribes dénonçant tantôt les pratiques des pays en développement jugées attentatoires à la propriété intellectuelle, tantôt les comportements monopolistiques des multinationales de la pharmacie perçus comme préjudiciables à la santé des populations au Sud. Il importe donc d'énoncer les termes de l'ADPIC, les obligations et droits incombant aux pays membres de l'OMC au regard de la protection à la fois du brevet et de la santé publique.

\section{Objectifs et principes de l'ADPIC}

L'ADPIC est issu d'un cycle de négociations multilatérales qui a duré huit ans et s'est achevé en avril 1994 avec la création de l'OMC. Tout pays membre de l'OMC doit se soumettre aux règles de cet accord et s'expose à défaut à des sanctions commerciales.

L'OMC spécifie en premier lieu que l'objectif de l'ADPIC est de mettre en œuvre des standards internationaux minimums concernant la protection de la propriété intellectuelle ${ }^{3}$. Autrement dit, l'accord ne vise nullement à instituer un régime de

3 - Organisation mondiale du commerce, Accord sur les aspects des droits de propriété intellectuelle touchant au commerce, 1994, disponible sur www.wto.org. Voir aussi H. Desterbecq et B. Remiche, "Les brevets pharmaceutiques dans les accords du GATT: l'enjeu?", Revue internationale de droit 


\author{
DPI unique et universel; les membres pourront notamment adopter un régime \\ plus contraignant que celui prévu dans l'ADPIC (Article 1).
}

\begin{abstract}
Cet objectif ne constitue pas une "obligation absolue et unique» ${ }^{4}$. L'OMC reconnaît la nécessité pour les États membres de satisfaire des objectifs de développement et de santé publique. Le brevet doit «contribuer à la promotion de l'innovation technologique et au transfert et à la diffusion de la technologie, à l'avantage mutuel de ceux qui génèrent et de ceux qui utilisent des connaissances techniques et d'une manière propice au bien-être social et économique, et à assurer un équilibre de droits et d'obligations» (Article 7$)^{5}$.
\end{abstract}

économique, X (1), 1996, p. 7-68; P. Boulet et G. Velasquez, Mondialisation et accès aux medicaments : les implications des Accords ADPIC/OMC, Genève, Organisation mondiale de la santé, 1999; UNCTAD/ ICTSD, Resource Book on TRIPS and Development: an Authoritative and Practical Guide to the TRIPS Agreement, UNCTAD/ICTSD capacity building on IPRs, 2005; C. Mfuka, "Accord ADPIC et brevets pharmaceutiques - Le difficile accès des pays en développement aux médicaments anti-sida", Revue d'économie industrielle, $\mathrm{n}^{\circ} 99,2^{\mathrm{e}}$ trimestre, 2002, p. 191-214.

4 - P. Boulet et G. Velasquez, op. cit., 1999.

5 - On retrouve ici une première idée largement soutenue dans la littérature économique selon laquelle le brevet permet aux entreprises de recouvrer les dépenses colossales investies en recherche et développement (R\&D) lors de la mise au point de nouveaux médicaments. Dans cette perspective, le brevet est considéré à la fois comme le moteur de l'innovation et de l'amélioration du bien-être (voir F. Machlup, "An economic review of the patent system», Study No 15 of Commission on Judiciary, Sub comm. on Patents, Trademarks, and Copyrights, 85th Congress, 2d Session, 1958; K. J. Arrow, «Economic welfare and the allocation of resources for invention", in R. Nelson (éd.), The Rate and Direction of Inventive Activity, Princeton, Princeton University Press, 1962, p. 609-625; H. Demsetz, "Toward a Theory of Property Right», American Economic Review, vol.57, n² 2, mai 1967, p. 347-359). D’un autre côté, le brevet est décrit dans cet article 7 comme la pré-condition de transferts technologiques vers le Sud et de la fourniture aux pays en développement d'innovations techniques et thérapeutiques (voir E. Penrose, "International patenting and the less-developed countries», The Economic Journal, vol. 83, $n^{\circ} 331,1973$, p. 768-786; K.E. Maskus et J.H. Reichman, International Public Goods and Transfer of Technology under a Globalized Intellectual Property Regime, New York, Cambridge University Press, 2005; K. P. Gallagher, Putting Development First, Londres et New York, Zed Books, 2005). Ces deux arguments font l'objet de polémiques depuis deux siècles déjà. Concernant le premier point, il est rappelé que le brevet n'a jamais été aussi imposant dans les pays du Nord; pourtant le nombre de nouveaux médicaments ne cesse de décroître tandis que leurs prix n’ont jamais été aussi élevés dans les pays développés, spécialement aux États-Unis où le contrôle des prix est inexistant (voir F. R. Lichtenberg, "Are the benefits of newer drugs worth their costs? Evidence from the 1996 MEPS", Heath Affairs, vol. 20, $\mathrm{n}^{\circ}$ 5, septembre/octobre 2001, p. 241-251; National Institute for Health Care Management, "Changing patterns of pharmaceutical innovation», Rapport de recherche, États-Unis, mai 2002). Pour expliquer ce phénomène, il est indiqué que les dépenses de R\&D dissimulent des dépenses colossales en marketing qui ont peu à voir avec des objectifs d'innovation mais davantage avec une volonté des entreprises du secteur d'assurer à grand renfort de publicité onéreuse le succès commercial de leurs produits (Public Citizen, «Rx R\&D myths : the case against the drug industry's R\&D "scare card" ", Congress Watch, juillet 2001). Pour le second point, on se souvient que des pays comme l'Angleterre, la France, l'Allemagne, la Suisse ou le Japon ont assuré l'innovation et l'amélioration du bien-être à travers la fourniture de nouveaux médicaments sur la base d'un régime de propriété intellectuelle moins contraignant qu'il ne l'est actuellement au Nord. Et l'on continue de s'interroger sur l'effet possiblement néfaste du brevet sur l'innovation, sur le développement industriel et sur le bien-être des populations au Sud : dans la mesure où l'existence de brevets sur le médicament peut donner lieu à une hausse du prix des médicaments au Sud, elle conduit à un transfert de richesses des pays payeurs du Sud vers les producteurs/détenteurs de brevets du Nord; de même, le fait qu'une entreprise du Sud ne puisse produire le médicament qu'en versant des royalties au détenteur du brevet (au Nord), ce qu'elle facture en bout de course au patient, constitue aussi un transfert de richesses des pays du Sud vers les pays du Nord. 
Il s'ensuit que les États membres peuvent légiférer de façon à sauvegarder «la santé publique [...] et l'intérêt public dans des secteurs d'une importance vitale pour leur développement socioéconomique et technologique» (Article 8.1). De même, ils peuvent prendre «des mesures appropriées" pour «éviter l'usage abusif des droits de propriété intellectuelle par les détenteurs de droits ou le recours à des pratiques qui restreignent de manière déraisonnable le commerce ou sont préjudiciables au transfert international de technologie» (Article 8.2). L'ADPIC n'est donc pas régi par un principe de protection inconditionnelle du brevet.

L'accord circonscrit le champ de la brevetabilité. Il établit qu' « un brevet pourra être obtenu pour toute invention, de produit ou de procédé, dans tous les domaines technologiques». Il n'est donc plus possible pour un pays d'exclure des produits ou des secteurs industriels du champ du brevet. Du reste, l'accord spécifie que les produits et procédés doivent être "nouveaux», impliquant "une activité inventive» et «susceptibles d'application industrielle» (Article 27.1).

De plus, en vertu du principe de traitement national (Article 3) qui interdit toute discrimination entre acteurs nationaux et non nationaux, l'usage d'un brevet doit être rendu possible que les produits soient «importés ou [...] d'origine nationale». La notion d'usage effectif du brevet (ou working patent) est donc rendue impraticable. Celle-ci prévoyait l'octroi d'un brevet sous condition d'une exploitation effective sous la forme d'une production locale. Disposition largement utilisée par le passé dans les pays développés puis dans les pays en développement pour encourager les transferts technologiques.

A contrario, des critères d'exclusion de la brevetabilité sont précisés notamment "pour protéger l'ordre public ou la moralité» ou "pour éviter de graves atteintes à l'environnement» (Article 27.2).

Les brevets pour les procédés de fabrication et les produits sont valables pour au moins vingt ans (Article 33). Cette disposition n'étant pas rétroactive, seuls les médicaments brevetés par les fabricants après 1995 peuvent faire l'objet d'une demande de brevet dans les pays membres de l'OMC.

Ainsi, obligation est faite aux pays membres de l'OMC d'assurer la protection de la propriété intellectuelle. Cependant, le principe de protection de la santé publique donne lieu à des exceptions aux droits conférés par les brevets : au nom de ces flexibilités, les brevets peuvent être contournés dans des circonstances particulières.

\section{Flexibilités et promotion}

de la santé publique sous ADPIC

Tandis que l'ADPIC est entré en vigueur en 1995 dans les pays développés, les pays en développement et les pays en transition vers une économie de marché ont bénéficié d'une période de grâce dite de transition (Article 65). La fin de cette période a été fixée à 2005 pour des pays émergents comme l'Inde, et à 2015 pour les pays les moins avancés. Toutefois, ces derniers pourront demander une extension de 
cette période en considération de leurs «besoins et impératifs spéciaux» et de leur «besoin de flexibilité pour se doter d'une base technologique viable» (Article 66.1). Ainsi, les pays africains par exemple devront se mettre en conformité avec l'ADPIC au plus tard en 2015 si la période de transition n'est pas prolongée.

En vertu des principes de l'ADPIC, un pays peut contourner le brevet pour promouvoir des objectifs de santé publique. "Dans des situations d'urgence nationale ou d'autres circonstances d'extrême urgence», "en cas d'utilisation publique à des fins non commerciales " (Article $31 b)^{6}$ ou de pratique avérée anti-concurrentielle de la part des fabricants de médicaments (Article 31c), un pays peut utiliser les droits conférés par un brevet sans l'autorisation de son détenteur ${ }^{7}$. Celui-ci doit simplement être informé dans un délai raisonnable et obtenir une compensation financière adéquate ${ }^{8}$. Ainsi, en cas d'épidémie de VIH/sida, si un médicament est commercialisé à prix prohibitif ou en quantité insuffisante pour un médicament, un pays pourra délivrer ce qu'on appelle une «licence obligatoire». Une agence publique ou une entreprise privée sera alors autorisée à produire le médicament pour traiter l'urgence nationale ou pour remédier à des pratiques monopolistiques de la part des fabricants: des médicaments génériques plus abordables seront rendus disponibles. L'accord prévoit que la définition d'une urgence nationale reste à la discrétion des États.

Dans les mêmes circonstances, un pays peut procéder à des importations parallèles, c'est-à-dire importer un médicament depuis un pays où il est commercialisé à un prix moindre. Toutefois, cette possibilité légale dépend du principe d'exhaustivité des droits retenu par le pays, le mieux étant le principe d'exhaustivité internationale. Suivant ce principe, le pays pourra importer de n'importe quel point du globe un médicament breveté commercialisé à un prix plus abordable. Au contraire, un principe d'exhaustivité nationale interdit à un pays de procéder à de telles importations parallèles ${ }^{9}$. En la matière, l'ADPIC reste muet : "aucune disposition du présent accord ne sera utilisée pour traiter la question de l'épuisement des droits de propriété intellectuelle»(Article 6). Les membres ont donc toute

6 - Travailler sur une molécule dans le cadre d'un programme de recherche ou produire le médicament pour le mettre gratuitement à disposition des populations dans le cadre d'un programme de santé publique ne constituent pas une violation des droits conférés par le brevet. Il n'y a pas exploitation commerciale, donc légalement pas de préjudice pour le détenteur du brevet.

7 - Il n'est pas indispensable de chercher à obtenir auprès du détenteur du brevet une licence volontaire, c'est-à-dire le transfert volontaire des droits contre paiement de royalties fixées par négociations.

8 - On parle alors de "compensation", prevue ou non par la loi, ce qui est une manière de reconnaître le préjudice subi par le détenteur du brevet, dont on exploite le medicament sans son autorisation, sous licence dite "obligatoire».

9 - Dans le dernier cas d'un principe d'exhaustivité régionale, les pays d'importation et d'exportation du médicament devront appartenir à la même organisation régionale : l'Union européenne, l'Organisation africaine de la propriété intellectuelle ou encore l'African Regional Industrial Property Organization (organisations de protection de la propriété intellectuelle couvrant respectivement les zones francophone et anglophone d'Afrique subsaharienne). 
latitude pour définir le principe retenu et préciser l'étendue du recours possible aux importations parallèles ${ }^{10}$.

\section{La déclaration ministérielle de Doha : \\ la santé publique avant la protection du brevet}

Face aux difficultés et aux pressions subies par les pays en développement dans l'usage pratique des flexibilités prévues par l'ADPIC ${ }^{11}$, les États membres de l'OMC ont dû réitérer leur attachement au principe de protection du brevet en tant qu'incitation à l'innovation lors de la réunion lançant le cycle de Doha en 2001 : «la protection de la propriété intellectuelle est importante pour le développement de nouveaux médicaments» (paragraphe 3 de la déclaration ministérielle de Doha). Mais ils ont dû également réaffirmer le principe selon lequel la protection des brevets est subordonnée à la santé publique: "nous convenons que l'accord sur les ADPIC n'empêche pas et ne devrait pas empêcher les membres de prendre des mesures pour protéger la santé publique». Par conséquent, "nous affirmons que ledit accord peut et devrait être interprété et mis en œuvre d'une manière qui conforte le droit des membres de l'OMC de protéger la santé publique et, en particulier, de promouvoir l'accès de tous aux médicaments» (paragraphe 4) ${ }^{12}$.

Fut donc réaffirmée la possibilité pour les pays membres de recourir aux licences obligatoires et aux importations parallèles dans le cas notamment d'une urgence nationale, et d'avoir toute autorité pour définir ce qui constitue une urgence nationale (paragraphes $5 b$ et $5 c$ ). En outre, il fut également rappelé que les membres sont libres d'adopter leur principe d'exhaustivité des droits et de spécifier en conséquence l'étendue du recours aux importations parallèles (paragraphe $5 d$ ). Toutefois, devant les objections émises par les pays africains, qui étaient dans l'impossibilité d'accorder des licences obligatoires faute de capacités de production domestiques suffisantes, les États membres ont été chargés de trouver une solution à ce problème dans les deux ans (paragraphe 6).

En août 2003, quelques mois avant le sommet de Cancun, un accord a été trouvé et une flexibilité additionnelle introduite: les pays membres ont la possibilité de procéder à des importations sous licence obligatoire. Ainsi, un pays dépourvu

10 - Cependant, certains rappellent qu'un principe d'exhaustivité internationale pourrait inciter les entreprises à opter pour un prix unique dans le monde pour mettre fin aux importations parallèles. En effet, les firmes peuvent être soucieuses de prévenir les effets indésirables d'exportations depuis des pays où les médicaments sont commercialisés à des prix préférentiels plus faibles vers des pays où les prix pratiqués sont plus élevés. La mise en œuvre d'un prix unique signifierait une révision considérable des prix à la hausse dans les pays en développement et une sérieuse dégradation de l'accessibilité des traitements. Ce faisant, il est recommandé aux membres de retenir un principe d'exhaustivité nationale. D'autres s'opposent vivement à cet argument car renoncer aux importations parallèles serait renoncer à la possibilité d'introduire plus de concurrence sur un marché et de tirer le prix des médicaments à la baisse.

11 - Dues en grande partie aux imprécisions et ambiguïtés contenues dans certaines dispositions.

12 - Organisation mondiale du commerce, "Déclaration sur l'accord ADPIC et la santé publique», déclaration ministérielle, Conférence ministérielle de l'OMC, Doha, 14 novembre 2001, disponible sur www.wto.org. 
de capacité de production peut accorder une licence obligatoire à une entreprise installée dans un pays tiers et lui demander de produire et d'exporter vers son territoire des médicaments pour lui permettre de traiter une urgence sanitaire. Pour ce faire, des conditions ont été établies : déterminer au préalable le volume de production requis, identifier sans équivoque les produits fabriqués, notifier le pays de destination ou encore accorder une compensation financière adéquate au détenteur du brevet ${ }^{13}$.

Ainsi, des flexibilités existent et, devant les besoins sanitaires criants du Sud, certains pays explorent ces flexibilités pour promouvoir l'accès aux médicaments vitaux. C'est le cas du Brésil, de la Thaïlande et de l'Inde.

\section{Promotion de la santé publique \\ et accessibilité des médicaments anti-sida au Sud : les cas brésilien, thaillandais et indien}

Le Brésil et la Thaïlande ont mis en place des politiques publiques d'accès aux antirétroviraux en exploitant la possibilité de recourir aux licences obligatoires. Parallèlement, l'Inde explore le champ d'une brevetabilité limitée pour prévenir l'octroi de monopoles temporaires pour des médicaments jugés peu innovants : des objectifs à la fois sanitaires et industriels sont ici visés. Hélas, l'usage de ces flexibilités est trop souvent à l'origine d'accusations virulentes source de confusions.

\section{De l'usage avisé des licences obligatoires : la posture brésilienne}

Le Brésil fut le premier pays du Sud à mettre en place un programme d'accès universel aux antirétroviraux. Le programme débute en 1997 avec la fourniture gratuite de traitements à plus de 20000 patients infectés par le VIH/sida ${ }^{14}$. Le budget annuel alloué à l'achat des médicaments anti-sida est alors de 224 millions de dollars. Cette même année, le Brésil renonce à la période de transition prévue

13 - Concernant la diffusion de l'innovation et les transferts technologiques vers les pays en développement, autres objectifs étroitement associés à l'obligation de protection de la propriété intellectuelle, force est de constater que les dispositions concrètes manquent en comparaison des outils déployés pour assurer la protection des brevets. Certes, l'ADPIC note que «les pays développés membres offriront des incitations aux entreprises et institutions sur leur territoire afin de promouvoir et d'encourager le transfert de technologie vers les pays membres les moins avancés pour leur permettre de se doter d'une base technologique solide et viable» (Article 66.2). De même, la déclaration de Doha convient «d'un examen, au sein d'un Groupe de travail sous les auspices du Conseil général, de la relation entre commerce et transfert de technologie, et de toutes recommandations éventuelles sur les mesures qui pourraient être prises dans le cadre du mandat de l'OMC pour accroître les apports de technologie aux pays en développement» (paragraphe 37). Il reste qu'avec l'interdiction faite aux pays membres de mettre en œuvre ou de conserver la notion d'usage effectif d'un brevet sous la forme d'une production locale, il semble que le seul outil qui aurait pu soutenir la diffusion de transferts technologiques et de l'innovation vers le Sud ait disparu.

14 - P. Chequer, "Access to treatment and prevention : Brazil and beyond», rapport, ministère de la Santé brésilien, 2007, disponible sur www.saude.gov.br. 
par l'ADPIC jusqu'en 2005. Le pays amende sa loi et introduit le brevet sur le médicament.

En 2001, le nombre de personnes placées sous traitement passe la barre des 100000. À cette date, sur douze médicaments distribués gratuitement, huit sont produits localement. Far-Manguinhos, unité publique de recherche et de production, peut librement réaliser la fabrication de la plupart de ces antirétroviraux non brevetés au Brésil. Ils ont été découverts avant 1995, date d'entrée en vigueur de l'ADPIC. Le budget alloué à l'achat des médicaments est de 232 millions de dollars. Par ailleurs, les autorités sanitaires observent une résistance croissante des patients aux traitements de première intention ${ }^{15}$ composés de médicaments non brevetés. Par conséquent, la mise sous traitement de seconde intention s'impose, requérant cette fois-ci la prescription de médicaments brevetés. C'est le cas de l'Efavirenz, médicament breveté par Merck, qui absorbe à lui seul 10 \% du budget antirétroviral.

Le Brésil craint que le recours grandissant à des médicaments brevetés ne menace la viabilité de son programme d'accès universel aux rétroviraux, programme qui permet aux patients de vivre avec la maladie, de réduire le nombre d'hospitalisations et d'économiser finalement des sommes considérables. Aussi, le pays tentet-il d'obtenir des réductions de prix importantes : il mobilise les outils légaux disponibles pour négocier avantageusement avec les entreprises détentrices des brevets.

La première offensive brésilienne concerne l'Efavirenz. En mars 2001, Merck menace de poursuivre le Brésil pour importation illégale de génériques de l'Efavirenz, pratique qui constituerait un viol qualifié de son brevet. La réponse brésilienne est immédiate. Far-Manguinhos a effectivement procédé à des importations de génériques indiens. Ces importations n'ont pas pour but une exploitation commerciale. L'unité publique de recherche et de production n'a nullement l'intention de les vendre sur le marché. En réalité, Far-Manguinhos travaille sur des échantillons du générique indien pour développer et produire à terme sa propre copie. Pratique parfaitement légale puisque l'ADPIC autorise le contournement du brevet en cas d'usage gouvernemental et non commercial : un programme de R\&D sur un médicament et/ou la production du générique d'un médicament breveté sous licence obligatoire pour approvisionner un programme d'accès universel aux médicaments essentiels ne constituent pas une violation de la propriété intellectuelle.

Si, dans un premier temps, Merck rappelle que des réductions de prix sont accordées aux pays les plus pauvres où le taux d'infection est élevé (plus de $1 \%$ de la population totale), la menace d'une production locale brésilienne trouve très vite un écho favorable. L'entreprise propose de réduire de $65 \%$ le prix de l'Efavirenz qui passe de 2,09 à 0,84 dollar le comprimé. D’où une économie annoncée de 39 millions de dollars pour le ministère de la Santé brésilien.

15 - Un traitement de première intention est le premier traitement prescrit à un patient. Un traitement de seconde intention intervient lorsque le patient développe des résistances au premier traitement, ce qui se produit en moyenne dans $20 \%$ des cas pour le VIH/sida. 
Quelques mois plus tard, le Brésil s'attaque au Nelfinavir, médicament breveté par Roche. Après six mois de négociations infructueuses avec Roche, le gouvernement brésilien annonce son intention de passer outre le brevet. En décembre 2001, le contrat d'approvisionnement entre Roche et le ministère de la Santé brésilien arrive à terme. Il ne sera pas renouvelé et Far-Manguinhos sera chargé de procéder à la production locale du médicament. Une fois de plus, la procédure est légale puisqu'il s'agit d'une utilisation publique à des fins non commerciales, d'une production sous licence obligatoire destinée à alimenter le programme d'accès universel aux rétroviraux. Dans la mesure où un quart des 100000 patients du programme sont sous Nelfinavir, la production locale devrait permettre de réduire le prix du médicament de $40 \%$, soit une économie de 88 millions de dollars.

Très vite, le ministère lance la procédure de licence obligatoire pour entamer la production locale et l'importation de génériques indiens en attendant que FarManguinhos puisse assurer la montée en puissance de sa production pour couvrir les besoins domestiques. Roche estime que cette annonce constitue une interruption brutale des négociations en cours et rappelle que des dons importants de médicaments pédiatriques ont été accordés au programme de santé brésilien. Finalement, l'intention brésilienne de produire localement le Nelfinavir sous licence obligatoire conduit Roche à proposer une baisse de $35 \%$ du prix de son médicament. Un accord est finalement signé fin août : la réduction sera de $40 \%$, et Roche s'engage à entamer une production locale au plus tard en 2002. Le Brésil interrompt alors la procédure de licence obligatoire.

En 2003, le programme d'accès universel aux antirétroviraux couvre près de 140000 personnes. Des économies importantes ont été réalisées grâce à des négociations ardues avec les multinationales de la pharmacie. Le budget antirétroviral est passé de 232 millions de dollars à 181 millions entre 2001 et 2003, bien que le nombre de patients ait augmenté de $40 \%$. Toutefois, le ministère brésilien s'inquiète du fait que trois antirétroviraux brevetés absorbent $63 \%$ de son budget médicament: l'Efavirenz, le Nelfinavir et le cocktail Lopinavir/Ritonavir ${ }^{16}$. En particulier, le ministère constate une augmentation sensible du nombre de patients en demande de Lopinavir/Ritonavir et le prix élevé de ce cocktail anti-sida.

Sans surprise, le Brésil entame des négociations avec les entreprises détentrices des brevets pour obtenir une réduction d'au moins $40 \%$ du prix de ces médicaments. Parallèlement, le gouvernement annonce son intention de réaliser production locale et importations de génériques. Du côté des entreprises, Merck rappelle que le prix de l'Efavirenz a été réduit de 85 \% ces dernières années pendant qu'Abbott refuse de vendre son cocktail Lopinavir/Ritonavir au prix plus faible réservé aux pays les plus pauvres. Le Brésil insiste en indiquant qu'une production locale sous licence obligatoire ou l'importation de génériques ramènerait le prix du Lopinavir/

16 - Un cocktail est un traitement composé de plusieurs médicaments, le plus souvent deux ou trois, se présentant sous la forme d'un comprimé à prendre une à deux fois par jour. Cela permet de réduire la prise de médicaments, d'améliorer l'observance des patients et de prévenir finalement les risques de résistance. 
Ritonavir à 0,25 dollar le comprimé contre 1,5 dollar actuellement. De même, le prix de l'Efavirenz pourrait passer à 0,9 dollar contre 2,10 dollars sous princeps ${ }^{17}$. Les baisses de prix proposées avoisinent alors les $7 \%$. On est bien loin des $40 \%$ réclamés par le ministère de la Santé brésilien.

En 2005, le programme d'accès universel aux traitements anti-sida couvre 180000 patients pour un budget de près de 400 millions de dollars. Entre 1997 et 2005, le nombre de patients mis sous traitement a été multiplié par neuf alors que le budget n'a pas doublé. Pourtant, les ressources allouées à l'achat de médicaments sous brevet ont fortement progressé : près de $79 \%$ du budget médicament, avec une facture de 310 millions de dollars. En somme, cette prouesse tient à la capacité du Brésil à négocier avec les multinationales du médicament.

Actuellement, le Brésil cherche à alléger la contrainte financière qu'exerce l'Efavirenz sur son budget médicament : près de $38 \%$ des patients de son programme de santé publique prennent de l'Efavirenz, d'où une dépense de 55 millions de dollars par an, soit $14 \%$ de son budget médicament. Le pays a donc relancé les négociations avec Merck et réclame des baisses de prix de l'ordre de $60 \%$. À toutes fins utiles, il est rappelé que le générique indien est proposé à 0,45 dollar le comprimé contre 1,56 dollar le princeps, ce qui permettrait une économie de 30 millions par an. En d'autres termes, le Brésil demande à Merck de lui vendre le médicament au prix proposé dernièrement au gouvernement thaïlandais (voir plus bas). L'entreprise a fait savoir aux autorités sanitaires que douzième puissance économique mondiale, le Brésil affiche un PIB par tête plus élevé que celui de la Thaïlande et ne peut bénéficier d'un tel prix préférentiel.

L'affaire est en cours, mais déjà le Brésil observe avec anxiété que les baisses de prix commencent à se tasser et s'inquiète d'un essoufflement de son pouvoir de négociation. Le pays s'interroge sur la façon de réactiver ce pouvoir et de relancer les baisses de prix pour assurer la perennité de son programme d'accès universel aux antirétroviraux.

\section{Persévérance et endurance à la pression internationale : la posture thaïlandaise}

Dans les années 1990, sous la pression des États-Unis, la Thaïlande amende sa loi sur le brevet et instaure un régime de propriété intellectuelle plus contraignant, deux ans avant la ratification de l'ADPIC $^{18}$. Avant 1992, la loi thaillandaise ne reconnaissait les brevets que sur les procédés et autorisait donc les firmes locales à copier en toute légalité les médicaments brevetés ailleurs ${ }^{19}$. Après cette date, la

17 - Un princeps est un médicament de marque, c'est-à-dire produit par la firme détentrice du brevet.

18 - Voir S. Guennif et C. Mfuka, «Promesse et risque du renforcement du brevet au Sud. Transfert technologique, développement pharmaceutique et accès aux traitements», Cahiers de l'association Tiers Monde, $\mathrm{n}^{\circ} 20$, «Droits et développement», 2005.

19 - Sur la base de la rétro-ingénierie (activité qui consiste à disséquer une molécule et à en définir les caractéristiques internes en partant du produit fini), le brevet sur les procédés autorise 
loi est révisée afin d'introduire les brevets sur les produits. Désormais, tous les médicaments inventés après 1992 sont brevetables en Thaïlande pour une durée de vingt ans, période durant laquelle aucune version générique ne peut être fabriquée et commercialisée. En 1999, la loi est de nouveau amendée pour une mise en conformité avec l'ADPIC. Cela permet notamment au pays de retirer certaines provisions allant bien au-delà des standards de l'OMC, des dispositions dites "ADPIC-plus»: le recours aux licences obligatoires et aux importations parallèles redevient moins contraignant.

Alors que l'épidémie du VIH/sida progresse, la Thaïlande éprouve à partir de 1999 les plus grandes difficultés à assurer la fourniture de médicaments abordables, comme l'illustre le cas de la Didanosine, antirétroviral dont le brevet, détenu par Bristol-Myers \& Squibb, date de 1987. La Government Pharmaceutical Organization (GPO), unité publique chargée de la production de médicaments destinés aux hôpitaux publics, est parvenue peu avant la signature de l'ADPIC à développer une version générique de la Didanosine. En 1998, Bristol-Myers \& Squibb dépose une demande de brevet pour une formulation améliorée de la Didanosine ${ }^{20}$. Elle obtient un brevet et la GPO n'a plus la possibilité de produire de génériques. Le médicament est alors commercialisé par Bristol-Myers \& Squibb à un prix prohibitif : 2,5 dollars le comprimé, alors que le salaire moyen quotidien en Thaïlande avoisine les 3,84 dollars.

La GPO demande qu'une licence obligatoire lui soit délivrée. Sous la pression des États-Unis ${ }^{21}$, le gouvernement thaïlandais renonce et interrompt la procédure de licence obligatoire. La GPO s'entête et propose finalement une nouvelle formulation : de la Didanosine en poudre qui ne constitue pas une violation du brevet de Bristol-Myers \& Squibb pour sa formulation améliorée. En 2001, les activistes ont porté plainte devant les tribunaux contre Bristol-Myers \& Squibb et ont réclamé le retrait du brevet accordé à l'entreprise pour manque d'activité inventive significative $^{22}$. Le but est d'obtenir que la GPO puisse produire la Didanosine en comprimé, formulation plus confortable pour les patients et moins chère. Le brevet n'a pas été

les entreprises à innover autour de la molécule et à développer un procédé de fabrication différent de celui que couvre le brevet détenu par l'entreprise à l'origine de l'innovation thérapeutique. A contrario, lorsque la loi autorise également les brevets sur les produits, il devient impossible pour tout fabricant de déposer un brevet sur un autre procédé de fabrication. Cette distinction entre brevet sur les procédés et brevet sur les produits constitue le cour des législations nationales tant au Nord qu'au Sud. Ajoutons que nombre de pays du Nord ont, dans les premières phases de leur développement industriel, opté pour un régime de brevet couvrant uniquement les procédés pour inciter à la rétro-ingénierie et assurer le développement d'une industrie pharmaceutique nationale performante. Autrement dit, les pays du Sud ne font que suivre les exemples probants de l'Angleterre, de la France, de l'Allemagne, de la Suisse ou du Japon en matière de développement industriel (voir C. Mfuka, "Accord ADPIC et brevets pharmaceutiques...», art. cit., 2002).

20 - Cette amélioration réside dans l'association d'un anti-acide à la formulation antérieure.

21 - La Thaïlande est placée sous «Special 301 », instrument utilisé par les États-Unis pour exercer des pressions commerciales sur les pays qui portent atteinte à ses intérêts économiques en adoptant des standards jugés insuffisants pour la protection de la propriété intellectuelle.

22 - L'ajout d'un anti-acide constitue selon les activistes un cas d'innovation mineure réalisée autour d'un médicament déjà connu. Aussi, les critères d'inventivité requis par l'ADPIC ne sont pas respectés et la demande de brevet pour ce médicament n'aurait pas dû être satisfaite (voir plus bas). 
invalidé mais son étendue a été réduite, ce qui permet à la GPO de produire un comprimé de $100 \mathrm{mg}^{23}$. Le générique est alors commercialisé à la moitié du prix du princeps. Finalement, sous pression de la société civile, Bristol-Myers \& Squibb a annoncé qu'il renonçait à son brevet.

Cependant, depuis quelques mois, la Thaïlande est de nouveau sur la sellette et constitue la cible d'attaques des représentants du commerce des États-Unis. Ces derniers affirment que le pays viole les brevets des multinationales du médicament. À l'origine de ces attaques, la décision prise fin 2006 par les autorités thaïlandaises de délivrer des licences obligatoires pour des médicaments contre le VIH/sida et contre le cancer.

En février 2007, le ministère de la Santé thaïlandais publie un document dans lequel il répond point par point aux accusations des États-Unis. D'une part, il rappelle son droit légitime à user des flexibilités contenues dans l'ADPIC. D'autre part, il met en avant les efforts déployés pour négocier avec les entreprises détentrices des brevets et obtenir des prix plus abordables pour plusieurs médicaments essentiels. Précision importante puisque la procédure de délivrance d'une licence obligatoire pour urgence nationale n'exige pas que le pays entame au préalable des discussions avec les détenteurs des brevets; ceux-ci doivent simplement en être informés.

Dans ce document, il est précisé que le ministère de la Santé a mis en place en octobre 2003 un programme d'accès universel aux antirétroviraux couvrant depuis 80000 personnes infectées par le VIH/sida. Le budget de ce programme est passé de 10 à 100 millions de dollars entre 2001 et 2007, dont $20 \%$ proviennent du Fonds mondial de lutte contre le sida, la tuberculose et le paludisme, instance internationale placée sous l'autorité des Nations unies. Ce budget devrait augmenter de façon drastique dans les années à venir. En effet, les patients recevaient à l'origine des traitements de première intention composés essentiellement de médicaments brevetés dans la zone OMC avant 1995 mais non brevetés en Thailande, et donc disponibles sous formes génériques plus abordables. D'ici peu, $20 \%$ des patients présentant des résistances sévères à ces traitements seront mis sous traitements de seconde intention. Brevetables et brevetés en Thaillande, ces traitements ont un prix élevé faute d'une offre générique. C'est le cas notamment de l'Efavirenz et du cocktail Lopinavir/Ritonavir, recommandés par l'Organisation mondiale de la santé (OMS). Occasionnant une hausse importante des dépenses de santé, ce changement de thérapie pourrait compromettre la viabilité du programme d'accès universel aux rétroviraux.

Depuis deux ans, le gouvernement thaïlandais a entamé des négociations avec Merck et Abbott pour obtenir des baisses de prix. Devant l'échec des négociations, la Thaïlande a annoncé en novembre 2006, symboliquement quelques jours avant

23 - Oxfam, «Free trade agreement between the USA and Thailand threatens access to HIV/AIDS treatment», Oxfam Briefing Note, juillet 2004. 
la Journée mondiale de lutte contre le sida, son intention de délivrer une licence obligatoire pour cinq ans à la GPO pour assurer la production de l'Efavirenz et du cocktail Lopinavir/Ritonavir. Depuis, les réactions sont vives de la part des entreprises et des représentants du ministère du Commerce des États-Unis, qui tantôt accusent la Thaïlande de violer le contenu de l'ADPIC en ne respectant pas la propriété intellectuelle, tantôt estiment qu'il s'agit d'un coup bas des autorités qui n'ont pas cherché à négocier ${ }^{24}$.

Reste que depuis l'annonce de ces licences obligatoires, les négociations ont pris une autre tournure. En mars les menaces fusent: Abbott informe les autorités thaïlandaises qu'il ne commercialisera pas sept médicaments, dont une version plus adaptée pour les pays chauds du cocktail Lopinavir/Ritonavir. Puis, l'entreprise annonce en avril que le prix du cocktail sera réduit de moitié pour la Thaïlande et pour quarante autres pays en développement : il sera vendu à 1000 dollars par an et par patient contre 2200 dollars précédemment. Abbott précise que ce prix est inférieur à celui que proposent les fabricants indiens de génériques. De même, Merck propose de baisser le prix de l'Efavirenz : la bouteille passerait à 23 dollars.

Prolongeant le jeu des négociations, les autorités thaïlandaises insistent sur le fait que le Lopinavir/Ritonavir est actuellement vendu par Abbott à 500 dollars par an et par patient dans les pays les plus pauvres et que l'Efavirenz est vendu par les fabricants indiens de génériques à 20 dollars la bouteille. Du reste, les importations depuis l'Inde ont commencé en janvier en attendant que la GPO puisse, d'ici un an, produire le médicament en quantités suffisantes. Reste à savoir si la menace d'une licence obligatoire et le recours à des importations moins chères permettront à la Thaillande d'obtenir une baisse du prix de ces traitements assez conséquente pour réaliser 100 millions d'économies sur cinq ans et pour hausser à 100000 le nombre de patients sous traitement.

\section{De l'utilité d'une brevetabilité étroite du médicament : la posture sanito-industrielle indienne}

La trajectoire industrielle et sanitaire indienne est pleine d'enseignements pour les pays en développement. Suivant l'exemple historique des pays du Nord, l'Inde a amendé en 1970 sa loi sur le brevet dans le sens d'un assouplissement, comparé aux standards hérités de la période coloniale. Ce faisant, l'Inde a soutenu le développement d'une industrie pharmaceutique domestique performante, capable de fournir des médicaments plus abordables aux populations ${ }^{25}$. Forte de cette expérience,

24 - L'annonce faite de recourir aux licences obligatoires est intervenue quelques semaines après un coup d'État par lequel l'armée a démis le gouvernement en place. Cela a donné lieu à des pamphlets virulents dans la presse américaine. La Thaïlande y était qualifiée d'État voyou, pirate qui ne respecte pas ses engagements internationaux en matière de protection du brevet.

25 - Voir S. Guennif, «AIDS in India : public health related aspects of industrial policy and intellectual property rights in a developing country", CSH Occasional Paper 8, New-Delhi, Centre de sciences humaines, Publication of the French Research Institutes in India, 2004. Avant 1970, les brevets valent à la fois pour les procédés et les produits, ce qui oblige l'Inde à importer les médicaments produits par les multinationales et commercialisés à des prix prohibitifs. Après cette date, les brevets valent 
l'Inde entame tardivement l'amendement de sa loi sur le brevet après ratification de l'ADPIC; cela lui vaut des plaintes ou encore sa mise sous «Special 301 » à l'instar de la Thaïlande.

En 1999, un premier amendement de la loi sur le brevet est adopté pour satisfaire aux exigences de conformité rappelées par l'OMC ${ }^{26}$. Il prévoit le brevetage des médicaments. Les demandes de brevet sont donc déposées comme l'exige l'ADPIC et seront examinées en 2004 pour être éventuellement satisfaites en 2005. Les contrôleurs indiens devront s'assurer notamment que les demandes faites concernent bien des médicaments brevetés après 1995 dans la zone OMC. En 2002, un nouvel amendement de la loi apporte des précisions relatives au terme "invention», aux éléments non brevetables, aux droits du détenteur du brevet, à l'usage des licences obligatoires ou des importations parallèles.

Enfin, en 2005, l'Inde procède à un nouvel amendement de sa loi sur le brevet. Celui-ci vise tout d'abord à limiter les critères de brevetabilité : «la simple découverte d'une forme nouvelle d'une substance connue qui ne résulte pas d'une amélioration de l'efficacité connue de cette substance ou la simple découverte d'une nouvelle propriété ou d'un nouvel usage pour une substance connue [...] seront considérés comme la même substance, à moins qu'ils ne diffèrent de façon significative dans ses propriétés en considération de son efficacité» (Section 3 de l'Amendement Act de 2005). De cette façon, les nouveaux usages ou nouvelles indications thérapeutiques pour un médicament déjà connu ne seront pas systématiquement brevetables. En outre, une procédure d'opposition intervenant avant l'octroi d'un brevet est mise en place (Section 25). Enfin, il est prévu que les entreprises nationales qui ont réalisé des investissements significatifs dans la production et la commercialisation de certains médicaments avant l'entrée en vigueur de l'ADPIC en Inde seront autorisées à poursuivre leurs opérations. En échange, elles devront verser une compensation adéquate au tout nouveau détenteur du brevet ${ }^{27}$.

À l'évidence, en limitant la brevetabilité des innovations jugées mineures, et en profitant des flexibilités offertes par l'ADPIC, la posture indienne vise à concilier protection du brevet et promotion de la santé publique par l'accès des patients aux médicaments essentiels. De plus, des considérations industrielles sont perceptibles : l'Inde entend permettre à son industrie pharmaceutique de poursuivre son développement sur une base nationale large et ce faisant soutenir son positionnement sur un marché global dominé par les multinationales du Nord.

En se fondant sur des critères de brevetabilité retreints et grâce à la possibilité d'opposition précoce de toute entreprise à la demande de brevet déposée par un concurrent, les autorités indiennes ont rejeté en 2005 la demande déposée par

uniquement pour les procédés, ce qui permet à l'Inde de développer considérablement sa production nationale et conduit à une amélioration sensible de l'accessibilité des médicaments.

26 - Voir B. K. Keayla, "TRIPS patent system : core and important issues of concern", National Working on Patent Law, Governement of India, 45 p.

27 - «The Patents (Amendement) Act (2005)», Gazette of India, 7 avril 2005. 
Novartis pour son Glivec, traitement contre la leucémie. Elles ont jugé que le Glivec n'était pas un nouveau médicament mais la forme nouvelle d'un médicament déjà connu dont le brevet date de 1993. Contestant cette décision en faisant valoir que les critères de brevetabilité retenus n'étaient pas conformes aux dispositions prévues par l'ADPIC, Novartis a été dernièrement débouté une seconde fois par les autorités indiennes ${ }^{28}$.

De même, plusieurs organisations non gouvernementales (ONG) ont fait opposition à la demande de brevet déposée par Gilead pour son Tenofovir, médicament recommandé par l'OMS pour traiter le $\mathrm{VIH} /$ sida; elles discutent le caractère novateur de ce médicament. En contestant la demande de brevet, les ONG entendent soutenir l'accessibilité au médicament: elles craignent que son prix ne devienne prohibitif sous brevet et monopole temporaire. Dans le même temps, une procédure d'opposition a été engagée contre la demande de brevet de GlaxoSmithKline pour son Combivir, un cocktail anti-sida. Depuis, Gilead a annoncé son intention de céder des licences volontaires aux producteurs indiens pour le Tenofovir dès obtention d'un brevet en Inde ${ }^{29}$.

Par ailleurs, l'Inde a amendé sa loi sur le brevet de façon à autoriser les exportations de médicaments sous licence obligatoire. Ainsi, les entreprises indiennes devraient pouvoir produire des copies de médicaments brevetés et exporter des génériques moins chers vers les pays africains par exemple - sous réserve de satisfaire aux autres conditions réglementaires définies à Doha.

En somme, consciente de ses obligations vis-à-vis de l'OMC, l'Inde amende actuellement sa loi sur le brevet d'une façon qui lui permette de promouvoir la santé publique. Certes, l'industrie pharmaceutique indienne s'est illustrée en commercialisant des antirétroviraux génériques dans le monde, en exerçant une pression concurrentielle colossale sur les multinationales et en tirant le prix des traitements vers le bas. Cela a grandement contribué en bout de course à une amélioration de l'accessibilité des médicaments au Sud. Pour autant, on attend toujours le début d'un programme d'accès universel aux antirétroviraux en Inde. À ce jour, le mot d'ordre reste la prévention dans un pays où le facteur démographique fait craindre une progression de l'épidémie parmi la population générale et où les débats autour du nombre réel d'infections restent animés. En conséquence, il semble que le seul outil mis en place pour assurer l'accès des populations aux traitements, y compris pour les femmes enceintes infectées par le VIH/sida, reste le jeu du marché : une

28 - Selon Novartis, l'ADPIC dispose qu'«un brevet pourra être obtenu pour toute invention» (Article 27). À ce titre, toute substance pharmaceutique, qu'il s'agisse d'une "nouvelle molécule chimique» ou d'une "nouvelle entité médicale», doit pouvoir jouir d'un brevet. Or, l'accord ne définit pas le terme invention. Nulle part, il n'est pas fait mention du sens des termes «nouvelle molécule chimique» ou "nouvelle entité médicale», aussi l'interprétation prévaut en la matière: interprétation étroite du terme innovation (innovation majeure couvrant les nouvelles molécules chimiques) ou interprétation large (innovation mineure couvrant également les nouvelles formulations, combinaisons, indications thérapeutiques).

29 - J. Chaisse et S. Guennif, "L'économie politique du brevet au Sud : variations indiennes sur le brevet pharmaceutique», Revue internationale de droit économique, XXI, 2, 2007, p. 185-210. 
concurrence vive à l'origine des prix parmi les plus faibles dans le monde. Ajoutons enfin que l'Inde subit de nombreuses infections et épidémies, ce qui pose la question de la rareté des ressources disponibles pour les traiter et de l'équité de leur répartition entre différents objectifs de santé publique.

\section{Conclusion}

Assurément, l'ADPIC commande la protection du brevet pour les pays membres de l'OMC. Cependant, objectifs et principes rappellent que cette obligation ne doit pas empiéter sur les impératifs de santé publique des pays en développement, spécialement à un moment où progressent de nombreuses épidémies. S'il en était besoin, il a été montré ici que les flexibilités prévues par l'ADPIC sont plus que nécessaires à la protection de la santé publique et à l'accès des populations aux médicaments essentiels. L'exemple des antirétroviraux rappelle que l'enjeu est de taille au Sud en considération des obstacles que doivent surmonter les pays pour utiliser de façon effective ces flexibilités, qu'il s'agisse des licences obligatoires, des importations parallèles ou des critères de brevetabilité des médicaments.

Si l'usage de ces mesures reste indispensable pour assurer la promotion de la santé publique et contribuer au développement socioéconomique des pays du Sud, c'est qu'à ce jour rien ne s'est révélé être plus efficace pour activer la concurrence et améliorer l'accessibilité aux traitements. La médiatisation de l'épidémie du $\mathrm{VIH} /$ sida dans le monde et particulièrement en Afrique sub-saharienne a permis d'établir l'ampleur de la catastrophe sanitaire et l'existence de médicaments pour traiter l'infection. Or, le rapport de Médecins sans frontières ${ }^{30}$ sur le prix des antirétroviraux dans le monde a montré que ces médicaments étaient vendus à un tarif prohibitif jusqu'au début des années 2000, date d'entrée des fabricants indiens de génériques sur ce marché. Pour beaucoup, c'est la capacité de ces derniers à produire dès le début des années 2000 des antirétroviraux moins chers, voire plus confortables pour les patients (les premiers cocktails anti-sida furent proposés par ces fabricants), qui a permis d'améliorer l'accessibilité de traitements vitaux. Aussi, cette concurrence doit rester vive dans l'avenir, sous peine de voir le prix des nouveaux traitements devenir inaccessible au plus grand nombre, et la viabilité des programmes de santé publique compromise. D'où la nécessité d'un usage croissant des flexibilités prévues par l'ADPIC pour faire jouer la concurrence entre producteurs de princeps et fabricants de génériques.

Alors, il importe de pointer une autre difficulté pour les pays en développement, celle de ne pas sombrer dans les méandres d'un accord de libre-échange avec les États-Unis. La dizaine d'accords passés à ce jour entre les États-Unis et le Chili, la Jordanie, le Maroc, Singapour, l'Australie ou encore l'Amérique centrale (impliquant le Costa Rica, le Salvador, le Guatemala, le Honduras et le Nicaragua) ${ }^{31}$

30 - Médecins sans frontières, Untangling the Web of Price Reductions : a Pricing Guide for the Purchase of ARVs for Developing Countries, ge édition, juillet 2006.

31 - Et les accords en cours de négociation avec la Corée du Sud, l'Union douanière sud-africaine (composée de l'Afrique du Sud, du Botswana, de la Namibie, du Lesotho et du Swaziland), les 
promeut de façon offensive la protection du brevet dans le monde, circonscrit dangereusement le recours aux flexibilités prévues par l'ADPIC et limite le jeu de la concurrence générique ${ }^{32}$. Les clauses ADPIC-plus y sont en bonne place; en contrepartie, promesse est faite aux pays du Sud parties prenantes de ces accords d'un accès plus large aux marchés des États-Unis pour leurs produits agricoles notamment. À terme, la multiplication de ces accords de libre-échange pourrait miner la poursuite des objectifs de développement du Millénaire, et les efforts déployés par d'autres instances internationales (OMS, Fonds mondial...) et les autorités sanitaires nationales en général, pour promouvoir la santé publique et l'accessibilité des médicaments essentiels ${ }^{33}$.

Enfin, les exemples brésilien, thaïlandais ou indien exposés ici soulignent combien le recours effectif aux flexibilités prévues par l'ADPIC reste l'exception au Sud. Ils indiquent également que le soutien à la santé publique dans les pays du Sud passe moins par la fixation d'objectifs, de calendriers sanitaires multiples au sein des instances internationales, que par une posture préalablement ferme vis-à-vis de pratiques attentatoires aux droits et devoirs des pays du Sud en matière de protection de la santé publique.

pays andins (regroupant la Colombie, le Pérou, l'Équateur et la Bolivie) ou encore les Émirats arabes unis.

32 - Voir F.M. Abbott, Intellectual Property Provisions of Bilateral and Regional Trade Agreements in Light of US Federal Law, Issue Paper $\mathrm{n}^{\circ} 12$, International Centre for Trade and Sustainable Development/United Nations Conference on Trade and Development, Genève, 2006; S. Guennif, "One size fits all, but which one? From TRIPS agreement to TRIPS Plus agreements. Intellectual property rights regimes and access to medicines in developing countries», International Conference, DIME Workshop, "Intellectual property rights for business and society», Londres, 2006; F. Rossi, "Free trade agreements and TRIPS-plus measures", International Journal of Intellectual Property Management, vol. $1, \mathrm{n}^{\circ} 1$, p. $150-172$.

Pour certains pays en développement, la capacité politique à recourir effectivement aux flexibilités prévues par l'ADPIC est largement limitée par les sanctions commerciales diverses brandies par les pays du Nord, États-Unis en tête, ce qui réduit déjà le jeu de la concurrence générique.

33 - Ces accords devraient permettre aux États-Unis d'obtenir dans le cadre feutré d'accords bilatéraux et régionaux ce qui n'a pu être obtenu au sein de l'OMC. Entre autres, la protection des données cliniques (données utilisées jusqu'ici par les fabricants de génériques pour obtenir les autorisations de mise sur le marché de leurs produits), les limites opposées à l'usage effectif des importations parallèles ou encore l'absence de référence explicite à l'objectif assigné aux parties prenantes d'assurer la promotion de la santé publique sont autant de moyens qui devraient permettre aux multinationales du Nord de protéger avantageusement leur position de monopole, d'empêcher le jeu de la concurrence générique et d'altérer l'accessibilité des médicaments vitaux (voir S. Guennif, art. cit., 2006). 


\section{Bibliographie}

- Аввотт F. M., Intellectual Property Provisions of Bilateral and Regional Trade Agreements in Light of US Federal Law, Issue Paper $n^{\circ} 12$, International Centre for Trade and Sustainable Development/United Nations Conference on Trade and Development, Genève, 2006.

- Arrow K.J., "Economic welfare and the allocation of resources for invention", in R. Nelson (éd.), The Rate and Direction of Inventive Activity, Princeton, Princeton University Press, 1962, p. 609-625.

- Boulet P. et G. Velasquez, Mondialisation et accès aux médicaments : les implications des Accords ADPIC/OMC, Genève, Organisation mondiale de la santé, 1999.

- Chaisse J. et S. Guennif, «L'économie politique du brevet au Sud : Variations indiennes sur le brevet pharmaceutique», Revue internationale de droit économique, XXI, 2, 2007, p. 185-210.

- Chequer P., "Access to treatment and prevention: Brazil and beyond», Rapport, ministère de la Santé brésilien, disponible sur www.saude.gov.br., 2007

- Demsetz H., "Toward a theory of property right», American Economic Review, vol.57, $\mathrm{n}^{\circ} 2$, mai 1967, p. 347-359.

- Desterbecq H. et B. Remiche, «Les brevets pharmaceutiques dans les accords du GATT : l'enjeu?", Revue internationale de droit économique, X (1), 1996, p. 7-68.

- Drouhin N., V. TouzÉ et B. Ventelou, "Aids and economic growth in Africa: a critical assessment of the "base-case scenario" approach", in J.P. MOATTI, B. CORIAT, Y. BARnetT, Y. SouteYrand, J. Dumoulin et P.Y. FLORI (éds), Economics of Aids and access to HIV/AIDS Care in Developing Countries. Issues and Challenges, Paris, ANRS (Sciences sociales et Sida), 2003, p. 383-413.

- Gallagher K.P., Putting development first, Londres et New York, Zed Books, 2005.

- Gazette of India, "The Patents (Amendement) Act (2005)», 7 avril 2005.

- Guennif S., «One size fits all, but which one? From TRIPS agreement to TRIPS Plus agreements. Intellectual property rights regimes and access to medicines in developing countries", International Conference, DIME Workshop Intellectual Property Rights for Business and Society, Londres, 2006.

- GUENNIF S., "AIDS in India : public health related aspects of industrial policy and intellectual property rights in a developing country", CSH Occasional Paper 8, Centre de sciences humaines, New Delhi, Publication of the French Research Institutes in India, 2004.

- Guennif S. et C. Mruka, «Promesse et risque du renforcement du brevet au Sud. Transfert technologique, développement pharmaceutique et accès aux traitements", Cahiers de l'association Tiers Monde, $\mathrm{n}^{\circ} 20$, «Droits et développement», 2005.

- KeAYla B.K., «TRIPS patent system: core and important issues of concern", National Working on Patent Law, Governement of India, 2003, 45 p.

- Lichtenberg F.R., "Are the benefits of newer drugs worth their costs? Evidence from the 1996 MEPS", Heath Affairs, vol. 20, $\mathrm{n}^{\circ} 5$, septembre/octobre 2001, p. 241-251.

- Machlup F., "An economic review of the patent system", Study No.15 of Commission on Judiciary, Sub comm. on Patents, Trademarks, and Copyrights, 85th Congress, 2d Session, 1958.

- Maskus K. E. et J. H. Reichman, International Public Goods and Transfer of Technology under a Globalized Intellectual Property Regime, New York, Cambridge University Press, 2005.

- MÉdecins sans fRONTIÈres, Untangling the Web of Price Reductions : a Pricing Guide for the Purchase of ARVs for Developing Countries, $9^{e}$ édition, juillet 2006 .

- Mғuka C., "Accord ADPIC et brevets pharmaceutiques. Le difficile accès des pays en développement aux médicaments antisida", Revue d'économie industrielle, $\mathrm{n}^{\circ} 99$, $2^{e}$ trimestre 2002, p. 191-214.

- Ministère de la Santé publique thaïlandais et OfFice national de la sÉcurité sociale, "Facts and evidences on the 10 burning 
issues related to the government use of patents on three patented essential drugs in Thailand", Document to support strengthening of social wisdom on the issue of drug patent, février 2007.

- National institute for Health Care MANAGEMENT, «Changing patterns of pharmaceutical innovation», Rapport de recherche, États-Unis, mai 2002.

- ONUSIDA, Rapport sur l'épidémie mondiale de sida, édition spéciale $10^{\mathrm{e}}$ anniversaire de l'ONUSIDA, ONUSIDA/Programme commun des Nations unies sur le $\mathrm{VIH} / \mathrm{sida}$, 2006.

- Organisation mondiale du commerce, "Déclaration sur l'accord ADPIC et la santé publique», déclaration ministérielle, Conférence ministérielle de l'OMC, Doha, 14 novembre 2001, disponible sur www. wto.org.

- Organisation mondiale du commerce, "Accord sur les aspects des droits de propriété intellectuelle touchant au commerce», 1994, disponible sur www.wto.org.

- Oxfam, «Free trade agreement between the USA and Thailand threatens access to HIV/AIDS treatment», Oxfam Briefing Note, juillet 2004.

- Penrose E., «International patenting and the less-developed countries", The Economic Journal, vol. 83, ${ }^{\circ} 331,1973$, p. 768-786.

- Public Citizen, «Rx R\&D myths: the case against the drug industry's $R \& D$ "scare card" ", Congress Watch, juillet 2001.

- Rossi F., «Free trade agreements and TRIPS-plus measures", International Journal of Intellectual Property Management, vol.1, $\mathrm{n}^{\circ} 1,2006$, p. 150-172.

- UNCTAD/ICTSD, Resource Book on TRIPS and Development : an Authoritative and Practical Guide to the TRIPS Agreement, UNCTAD/ ICTSD capacity building on IPRs, 2005, disponible sur www.iprsonline.org/unctadictsd/ResourceBookIndex.htm. 